\title{
COMMENTARY
}

\section{Bioactive Lipids in Shoulder Tendon Tears}

Undurti N. Das

From UND Life Sciences, Battle Ground, Washington; and the BioScience Research Center, and Department of Medicine, Gayatri Vidya Parishad Medical College and Hospital, Visakhapatnam, India

A rotator cuff tear is a common cause of pain and disability among adults. It is estimated that each year, almost 2 million individuals in the United States have a rotator cuff problem. Current treatment options of rotator cuff tear include physiotherapy, administration of nonsteroidal anti-inflammatory drugs, local glucocorticoid injections, and surgery to repair torn tendons, all of which are not always effective.

There is reasonable evidence to suggest that rotator cuff tear is an inflammatory condition. But, current methods of investigation do not give adequate clues to predict who is likely to respond and recover from the treatment(s) offered and what factors control the local inflammatory and inflammation resolution process. Hence, understanding the cross talk among resident stromal cells, including fibroblasts that not only participate in inflammatory diseases of the joint but also in the switch from acute to chronic inflammation, tissue resident and infiltrating macrophages, infiltrating immune cells (that may include leukocytes and $\mathrm{T}$ cells), and endothelial cells, is important to the disease process and for the development of newer therapeutic interventions. In this context, the report by Dakin et $\mathrm{al}^{1}$ in this issue of The American Journal of Pathology is of substantial interest to the field. This report demonstrates that tendon stromal cells isolated from patients with tendon tears show a proinflammatory phenotype and secrete significantly higher amounts of IL-6 with dysregulated production and action of lipoxin A4 (LXA4), resolvins, protectins, and maresins compared with normal cells.

\section{Shoulder Tendon Tear Is an Inflammatory Condition}

The proinflammatory nature of shoulder tendon tears is supported by the reports that the mRNA expression levels of IL-1 $\beta$, IL-6, IL-8, tumor necrosis factor (TNF)- $\alpha$, transforming growth factor- $\beta 1$, cyclooxygenase- 2 (COX-2), matrix metalloproteinases (MMPs) 9, 1, and 13, and vascular endothelial growth factor are increased in their synovium and the concentrations of IL-1 $\beta$, IL-6, IL-8, TNF- $\alpha$, MMP-1, MMP-9, MMP-13, insulin-like growth factor- 1 , and transforming growth factor- $\beta 1$ are high in the synovial fluid. ${ }^{2,3}$ Furthermore, shoulder osteoarthritis that occurs as a result of degenerative rotator cuff tears seems to be related to persistent inflammation, oxidative stress, and angiogenesis, as evidenced by increased expression of angiopoietin 1 and 2 and hypoxia-inducible factor- $1 \alpha^{3}$ Immunohistochemical evaluation of torn supraspinatus tendon samples, obtained during arthroscopic shoulder surgery, showed a significantly increased number of macrophages, mast cells, and $\mathrm{T}$ cells compared with control. In addition, inflammatory cell infiltrate correlated inversely with rotator cuff tear size, with larger tears showing a marked reduction in all cell lineages, suggesting a role for innate immune pathways in the early tendinopathy. These results emphasize the fact that there are distinct and specific alterations in the expression of genes and their products that are involved in the process of inflammation and healing of the shoulder tendon tears.

During the acute phase of the shoulder tendon tear(s), infiltration of immune cells may help initiate the acute inflammatory process by up-regulating IL-1 $\beta$, IL-6, IL-8, TNF- $\alpha$, transforming growth factor- $\beta 1$, angiopoietins, insulinlike growth factor-1, hypoxia-inducible factor- $1 \alpha$, metalloproteinases, and COX-2 and its proinflammatory products, such as prostaglandin E2 (PGE2), thromboxanes (TXs), and leukotrienes (LTs). The production of these biologics is needed to induce the initial acute inflammatory process as inflammation is a protective phenomenon. It is likely that during this acute phase, there is increased production of proinflammatory molecules (such as IL-1 $\beta$, IL-6, IL-8, TNF- $\alpha$, and PGE2) and

Accepted for publication September 9, 2019.

Disclosures: None declared.

Address correspondence to Undurti N. Das, M.D., D.Sc., UND Life Sciences, 2221 NW 5th St, Battle Ground, WA 98604; and BioScience Research Centre and Department of Medicine, Gayatri Vidya Parishad Medical College and Hospital, Visakhapatnam 530048, India. E-mail: undurti@lipidworld.com 
relatively lower amounts of metalloproteinases, transforming growth factor- $\beta 1$, angiopoietins, insulin-like growth factor-1, hypoxia-inducible factor-1 $\alpha$, LXA4, and other antiinflammatory molecules. ILs and TNF- $\alpha$ activate phospholipase A2 (PLA2) and possibly other phospholipases that are needed for the release of arachidonic acid (AA; the precursor of PGE2, TXA2, LTs of 4 series, and LXA4); eicosapentaenoic acid [EPA; the precursor of prostaglandins (PGs) and TXs of 3 series, 5 series LTs, and resolvins of E series]; and docosahexaenoic acid (DHA; the precursor of resolvins of D series, protectins, and maresins) from the cell membrane lipid pool. ${ }^{3}$ It is noteworthy that IL-1 $\beta$ can enhance the production of IL-6 and nitric oxide (NO) in addition to its ability to augment PGE2 production. But blocking of NO synthesis in IL- $1 \beta$-stimulated cells enhances IL-6 production, suggesting a feedback regulation among IL-1, IL-6, and NO, with NO having an inhibitory action on IL-6 production. ${ }^{4}$ The infiltrating leukocytes, macrophages, $\mathrm{T}$ cells, and mast cells at the site of shoulder tendon tear(s) produce metalloproteinases in response to IL-1 $\beta$, IL- 6 , and TNF- $\alpha .{ }^{5}$ MMPs are needed for the release of TNF- $\alpha$ from macrophages and other cells, whereas TNF- $\alpha$ is essential for the induction of MMPs, which elicit macrophage infiltration. ${ }^{5}$ This cross talk is further influenced by the fact that TNF- $\alpha$ induces vascular endothelial growth factor production through the NF- $\kappa \mathrm{B}$ pathway. ${ }^{6}$ Neovascularization is essential to meet the energy needs of the cellular proliferation process for removal of the debris and proper healing. Yet, excess neovascularization may cause matrix accumulation and fibrosis that is usually seen in the degenerative cascade of rotator cuff tears.

P38 mitogen-activated protein kinases are a class of mitogen-activated protein kinases that respond to stress stimuli, such as cytokines, and are involved in cell differentiation, apoptosis, and autophagy. Cells, when exposed to IL-1 or TNF- $\alpha$ in the presence of p38 mitogen-activated protein kinase inhibition, showed decreased PGE2, NO, and IL-6 production and increased the ratio of tissue inhibitor of matrix metalloproteinase-1/MMP-3. These results suggest that inhibition of p38 mitogen-activated protein kinase can be used as a strategy to suppress inflammation.

\section{Essential Fatty Acid Metabolism and Shoulder Tendon Tear}

Dietary linoleic acid and $\alpha$-linolenic acid (which are essential fatty acids) are converted to their respective long-chain metabolites: $\gamma$-linolenic acid, dihomo- $\gamma$-linolenic acid, and AA from linoleic acid and EPA and DHA from $\alpha$-linolenic acid by the action of $\Delta^{6}$ and $\Delta^{5}$ desaturases and elongases. ${ }^{4}$ Dihomo- $\gamma$ linolenic acid is the precursor of prostaglandin E1, whereas AA and EPA form precursors to respective PGs, LTs, and TXs. PGs, LTs, and TXs have proinflammatory actions, although PGs formed from EPA have less proinflammatory actions compared with those formed from AA. ${ }^{4}$ Lipoxin A4, formed from AA and resolvins of $\mathrm{E}$ series from EPA and resolvins of
D series, protectins, and maresins, formed from DHA, have potent anti-inflammatory actions. ${ }^{4}$ Polyunsaturated fatty acids (PUFAs; linoleic acid, $\gamma$-linolenic acid, dihomo- $\gamma$-linolenic acid, AA, $\boldsymbol{\alpha}$-linolenic acid, EPA, and DHA) are released from the cell membrane lipid pool and form an important constituent of the cell membranes. It is likely that under physiological conditions, a balance is maintained between proinflammatory and anti-inflammatory products of $\mathrm{AA} / \mathrm{EPA} / \mathrm{DHA}$ and when this balance is tilted more toward proinflammatory metabolites, inflammatory events will be initiated. Thus, it is reasonable to propose that under conditions of increased PGE2, LT, and TX production, there could occur a decrease in the generation of LXA4 and other anti-inflammatory bioactive lipids (such as resolvins, protectins, and maresins) or they are metabolized to their less effective products. This implies that the way AA, EPA, and DHA are metabolized to form their products and the balance between the formed proinflammatory and antiinflammatory metabolites ultimately determine resolution of inflammation, wound healing, and restoration of homeostasis or continuation of inflammation and conversion of acute inflammation to its chronic stage.

In a previous study, Dakin et $\mathrm{al}^{8}$ demonstrated that tendonderived stromal cells obtained from healthy donors and patients with chronic tendinopathy, when treated with IL-1 $\beta$, showed markedly increased synthesis of PGs and proresolving mediators 15-epi-LXA4 and maresin R1. It was observed that healthy tendon cells, when treated with IL-1 $\beta$ in the presence of 15-epi-LXA4 or maresin R1, produced much less PGE2 and prostaglandin D2 (PGD2), whereas diseased cells showed significantly higher amounts of PGs, despite a significant decrease in IL-6 production. Furthermore, increased expression of 15-prostaglandin dehydrogenase (15-PGDH) with a concomitant increase in the concentrations of both 15-epiLXA4 and maresin R1 metabolites was noted in diseased tendon cells. Some of these abnormalities could be restored to normal by indomethacin (a COX-2 inhibitor) and a 15-PGDH inhibitor in diseased tendon cells in vitro. This, coupled with the report ${ }^{9}$ that in the early stage of inflammation genes and proteins induced by interferon and NF- $\mathrm{KB}$ are expressed, whereas in the late stage of inflammation (say in the chronic inflammation stage), genes and proteins are expressed by STAT- 6 and glucocorticoid receptor activation, is seen as rather interesting. These results imply that the initial acute inflammatory process is triggered by increased production of PGE2 and other proinflammatory molecules, such as LTs and TXs, with a concomitant production of LXA4, resolvins, protectins, and maresins (probably suboptimal); and as the resolution process sets in, this balance between proinflammatory and antiinflammatory molecules is tilted more toward the inflammation resolution inducing bioactive lipids: LXs, resolvins, protectins, and maresins. The failure of transition from the proinflammatory to anti-inflammatory events results in chronic pathologic inflammation. In this context, the interaction(s) among cytokines, glucocorticoids, and essential fatty acid metabolism, including the activities of COX-2 and 15-PGDH with regard to inflammation, is interesting. 


\section{Cross Talk among Cytokines, Glucocorticoids, and Bioactive Lipids}

PGE2 and LXA4 (and resolvins, protectins, and maresins) suppress IL-1 $\beta$, IL-6, TNF- $\alpha$, and metalloproteinase expression and, thus, suppress inflammation. In contrast, IL-1 $\beta$, IL-6, and TNF- $\alpha$ augment COX-2 expression and enhance the production of PGE2 and other proinflammatory eicosanoids. This paradoxical action of PGE2 to suppress IL-1 $\beta$, IL-6, and TNF- $\alpha$ production, yet promote inflammation, suggests that there is a cross talk between PGE2 and cytokines that is meant to fine-tune the inflammatory process. This also implies that there could be a dose- and timedependent interaction between PGE2 and inflammation. Blocking the activity of the prostaglandin degradation enzyme, 15-PGDH, results in a twofold increase in PGE2 levels with enhanced hemopoietic capacity and response to colon injury and partial hepatectomy in an accelerated manner, suggesting enhanced recovery from injury. ${ }^{10}$ Thus, PGE2 may have both proinflammatory and antiinflammatory actions and is needed for tissue repair and regeneration. AA is the precursor of both PGE2 and LXA4 and, hence, regulation of the AA metabolic pathway is crucial in the modulation of the inflammatory process. Inflammatory conditions in which increased PGE2 production is seen are also characterized by AA deficiency state and reduced production of LXA4. ${ }^{4,11}$ In such AA-deficient states, administration of AA enhanced LXA4 production with no change in PGE2 synthesis ${ }^{4,12,13}$ that could suppress inflammation. The increased PGE2 seen in acute inflammation may need to reach a critical level to trigger an antiinflammatory process to direct AA metabolism toward LXA4 synthesis.

Activation of PLA2 is needed to release PUFAs from the cell membrane lipid pool. There are two proposed phases of release of PUFAs: one at the onset of the generation of proinflammatory PGs, TXs, and LTs; and the other at the time of resolution for the synthesis of anti-inflammatory LXs and aspirin-triggered lipoxins, resolvins, protectins, and maresins. There are three classes of phospholipases that control the release of AA and other PUFAs: calcium-independent PLA2 (iPLA2), secretory PLA2 (sPLA2), and cytosolic PLA2 (cPLA2). ${ }^{4,11}$ During acute inflammation, TNF- $\alpha$ and other cytokines induce influx of neutrophils and other immunocytes concomitant with PGE2 and leukotriene B4 (LTB4) production. During the phase of resolution of inflammation, there is an increase in LXA4 (and possibly, resolvins, protectins, and maresins) and PGD2, and formation of its product 15deoxy $\Delta 12-14$ PGJ 2 occurs with a simultaneous decrease in PGE2 synthesis to stop neutrophil influx and enhance phagocytosis of debris. ${ }^{11,14,15}$ Thus, there are two waves of release of AA and other PUFAs: one at the onset of inflammation and the second at the time of resolution.

Studies revealed that type VI iPLA2 is the principal isoform expressed from the onset of inflammation up to 24 hours, whereas type IIa and V sPLA2 are expressed from the beginning of 48 to 72 hours. Type IV cPLA 2 is not detectable during the early phase of acute inflammation but increases progressively during resolution, peaking at 72 hours. The increase in type IV cPLA2 is mirrored by a parallel increase in COX-2 expression. ${ }^{11,15}$ This parallel increase in cPLA2 and COX-2 suggests a close enzymatic coupling between them. Selective inhibition of cPLA2 results in the reduction of PGE2, LTB4, and IL-1 $\beta$ (and platelet-activating factor), whereas inhibition of types IIa and V sPLA2 decreases platelet-activating factor and LXA4 (and possibly, resolvins, protectins, and maresins) with a concomitant reduction in cPLA2 and COX-2 activities. Thus, sPLA2-derived plateletactivating factor and LXA4/resolvins/protectins/maresins seem to induce COX-2 and type IV cPLA2. IL-1 $\beta$ induces cPLA2 expression, suggesting that IL-1 not only induces inflammation but also regulates cPLA2 expression to initiate resolution of inflammation. ${ }^{11,15}$ Glucocorticoids inhibit both cPLA2 and sPLA2 expression, whereas type IV iPLA2 expression is refractory to the steroids. In addition, steroids block the activities of $\Delta^{6}$ and $\Delta^{5}$ desaturases, COX-2, and lipoxygenase (LOX). Thus, steroids not only block the formation of PGE2, LTs, and TXs, but also induce an AA/EPA/ DHA deficiency state as a result of which adequate amounts of LXA4 (and possibly, resolvins, protectins, and maresins) cannot be formed and, hence, complete resolution of inflammation and wound healing would not occur because of their use. $^{11,15,16}$

Activated iPLA 2 contributes to the conversion of inactive pro-IL-1 $\beta$ to active IL-1 $\beta$, which, in turn, induces cPLA2 expression that is necessary for resolution of inflammation. TNF- $\alpha$ might have a direct suppressive action on the synthesis of LXs, PGD2, and 15deoxy $\Delta 12-14$ PGJ2, partly by its ability to suppress the activities of $\Delta^{6}$ and $\Delta^{5}$ desaturases that induce an AA/EPA/DHA deficiency state as a result of failure of conversion of linoleic acid and $\alpha$-linolenic acid to their respective long-chain metabolites. ${ }^{17}$ In contrast, LXA4/resolvins/protectins/maresins inhibit TNF$\alpha$-induced production of ILs; promote TNF- $\alpha$ mRNA decay and inhibit TNF- $\alpha$ secretion and leukocyte trafficking; and, thus, attenuate inflammation. This close interaction among PLA2s, COX-2, PGD2, LXA4, and platelet-activating factor in the initiation, maintenance, and resolution of inflammation suggests that any imbalance in this complex interplay may result in inappropriate inflammation and failure of its resolution and healing of wound.

Potassium leakage from cells activates iPLA2. In contrast, $\mathrm{Ca}^{2+}$-dependent phospholipase A2 (cPLA2) suppresses IL-1 $\beta$ processing. ${ }^{18}$ This, coupled with the report that potassium released by cells undergoing necrosis into the extracellular milieu causes suppression of T-cell effector function by suppressing Akt-mammalian target of rapamycin phosphorylation, ${ }^{19}$ indicates that potassium levels at the site of inflammation have a regulatory role in IL-1 $\beta$ formation, iPLA2 activation, and thus, modulation of inflammation. 


\section{Relationship between COX-2 and 15-PGDH}

The levels of PGE2 are controlled not only by the synthetic enzymes but also by the degrading enzyme. The key enzyme involved in degrading PGE2 is NAD ${ }^{+}$-dependent 15-PGDH, which catalyzes the oxidation of 15(S)-hydroxyl group of PGs and lipoxins to form their respective 15-keto-metabolites that have reduced biological activities. ${ }^{20}$ An increase in the expression of COX-2 and decrease of 15-PGDH may occur during inflammation, leading to an increase in the levels of PGE2 because cytokine-induced expression of COX-2 is accompanied by a down-regulation of 15-PGDH. Increased expression of 15-PGDH attenuates IL-1 $\beta$-induced expression of COX-2, suggesting an inverse relationship between COX-2 and 15-PGDH. ${ }^{20}$ In contrast, dexamethasone and other glucocorticoids induce the expression of 15-PGDH in a time- and dose-dependent manner that may explain their antiinflammatory actions. ${ }^{20}$ The expression of 15 -PGDH is positively related to mesenchymal markers and inversely to epithelial markers, implying that 15-PGDH is involved in epithelial-mesenchymal transition. ${ }^{20}$ This relationship between 15-PGDH expression and epithelial-mesenchymal transition may explain the possible role of PGE2 in the transition of acute inflammation to a chronic inflammatory state. Macrophages from nonobese diabetic mice have increased COX-2, low levels of 15-PGDH expression, and low levels of LXA4. This deficient LXA4 production is not due to deficient LOX activity but is related to increased soluble epoxide hydrolase, involved in metabolism of antiinflammatory epoxyeicosatrienoic acids. This suggests that chronic inflammation may be due to changes not only in COX-2 expression, but also as a result of abnormal activities of 15-PGDH and soluble epoxide hydrolase. ${ }^{21}$

\section{Conclusions and Therapeutic Implications}

Inflammation and its resolution are complex processes. The acute inflammatory response triggered by infection, injury, and other inciting agents either resolves in a timely manner or may become chronic because of an imbalance among various bioactive lipids. LXA4 (and other anti-inflammatory bioactive lipids, such as resolvins, protectins, and maresins) plays a pivotal role in suppressing an inappropriate inflammatory process and inducing much needed resolution, wound healing, and restoration of homeostasis. ${ }^{1}$ The sum effect or action of proinflammatory and anti-inflammatory bioactive lipids at the site of inflammation may be more important than the concentration of a single molecule. It is unclear whether local injection or infusion of LXA4 alone or a combination of LXA4 + resolvins + protectins + maresins is more beneficial at the site of inflammation, including rotator cuff tears. The possibility that oral supplementation (or local injection) of AA and other PUFAs in combination with nonsteroidal anti-inflammatory drugs and steroids may be of significant benefit in rotator cuff tears needs to be explored. Bioactive lipids have a regulatory role in stem cell proliferation and differentiation and bring about their beneficial action by elaborating LXA4 (and possibly, resolvins, protectins, and maresins). ${ }^{22}$ Future research may clarify whether local injections of LXA4 at the site of inflammation would stimulate stem cell proliferation and enhance wound repair.

\section{References}

1. Dakin SG, Colas RA, Wheway K, Watkins B, Appleton L, Rees J, Gwilym S, Little C, Dalli J, Carr JA: Proresolving mediators $\mathrm{LXB}_{4}$ and RvE1 regulate inflammation in stromal cells from patients with shoulder tendon tears. Am J Pathol 2019, 189:2258-2268

2. Millar NL, Hueber AJ, Reilly JH, Xu Y, Fazzi UG, Murrell GA, McInnes IB: Inflammation is present in early human tendinopathy. Am J Sports Med 2010, 38:2085-2091

3. Shindle MK, Chen CC, Robertson C, DiTullio AE, Paulus MC, Clinton CM, Cordasco FA, Rodeo SA, Warren RF: Full-thickness supraspinatus tears are associated with more synovial inflammation and tissue degeneration than partial-thickness tears. J Shoulder Elbow Surg 2011, 20:917-927

4. Poorani R, Bhatt AN, Dwarakanath BS, Das UN: COX-2, aspirin and metabolism of arachidonic, eicosapentaenoic and docosahexaenoic acids and their physiological and clinical significance. Eur J Pharmacol 2016, 785:116-132

5. Haro H, Crawford HC, Fingleton B, Shinomiya K, Spengler DM, Matrisian LM: Matrix metalloproteinase-7-dependent release of tumor necrosis factor-alpha in a model of herniated disc resorption. J Clin Invest 2000, 105:143-150

6. Ohba T, Haro H, Ando T, Wako M, Suenaga F, Aso Y, Koyama K, Hamada Y, Nakao A: TNF-alpha-induced NF-kappaB signaling reverses age-related declines in VEGF induction and angiogenic activity in intervertebral disc tissues. J Orthop Res 2009, 27:229-235

7. Studer RK, Aboka AM, Gilbertson LG, Georgescu H, Sowa G, Vo N, Kang JD: p38 MAPK inhibition in nucleus pulposus cells: a potential target for treating intervertebral disc degeneration. Spine (Phila $\mathrm{Pa}$ 1976) 2007, 32:2827-2833

8. Dakin SG, Ly L, Colas RA, Oppermann U, Wheway K, Watkins B, Dalli J, Carr AJ: Increased 15-PGDH expression leads to dysregulated resolution responses in stromal cells from patients with chronic tendinopathy. Sci Rep 2017, 7:11009

9. Dakin SG, Martinez FO, Yapp C, Wells G, Oppermann U, Dean BJ, Smith RD, Wheway K, Watkins B, Roche L, Carr AJ: Inflammation activation and resolution in human tendon disease. Sci Transl Med 2015, 7:311ra173

10. Zhang Y, Desai A, Yang SY, Bae KB, Antczak MI, Fink SP, Tiwari S, Willis JE, Williams NS, Dawson DM, Wald D, Chen WD, Wang Z, Kasturi L, Larusch GA, He L, Cominelli F, Di Martino L, Djuric Z, Milne GL, Chance M, Sanabria J, Dealwis C, Mikkola D, Naidoo J, Wei S, Tai HH, Gerson SL, Ready JM, Posner B, Willson JK, Markowitz SD: Inhibition of the prostaglandin-degrading enzyme 15-PGDH potentiates tissue regeneration. Science 2015, 348:aaa2340

11. Das UN: Current and emerging strategies for the treatment and management of systemic lupus erythematosus based on molecular signatures of acute and chronic inflammation. J Inflamm Res 2010, 3: $143-170$

12. Tateishi N, Kakutani S, Kawashima H, Shibata H, Morita I: Dietary supplementation of arachidonic acid increases arachidonic acid and lipoxin $\mathrm{A}_{4}$ contents in colon, but does not affect severity or prostaglandin $\mathrm{E}_{2}$ content in murine colitis model. Lipids Health Dis $2014,13: 30$

13. Tateishi N, Kaneda Y, Kakutani S, Kawashima H, Shibata H, Morita I: Dietary supplementation with arachidonic acid increases arachidonic acid content in paw, but does not affect arthritis severity or 
prostaglandin E2 content in rat adjuvant-induced arthritis model. Lipids Health Dis 2015, 14:3

14. Gilroy DW, Colville-Nash PR, Willis D, Chivers J, Paul-Clark MJ, Willoughby DA: Inducible cyclooxygenase may have antiinflammatory properties. Nat Med 1999, 5:698-701

15. Gilroy DW, Newson J, Sawmynaden P, Willoughby DA, Croxtall JD: A novel role for phospholipase A2 isoforms in the checkpoint control of acute inflammation. FASEB J 2004, 18:489-498

16. Croxtall JD, Choudhury Q, Tokumoto H, Flower RJ: Lipocortin-1 and the control of arachidonic acid release in cell signalling: glucocorticoids inhibit G protein-dependent activation of cPLA2 activity. Biochem Pharmacol 1995, 50:465-474

17. Mayer K, Schmidt R, Muhly-Reinholz M, Bögeholz T, Gokorsch S, Grimminger F, Seeger W: In vitro mimicry of essential fatty acid deficiency in human endothelial cells by TNF $\alpha$ impact of $\mathrm{w}-3$ versus w-6 fatty acids. J Lipid Res 2002, 43:944-951

18. Walev I, Klein J, Husmann M, Valeva A, Strauch S, Wirtz H, Weichel O, Bhakdi S: Potassium regulates IL-1 beta processing via calcium-independent phospholipase A2. J Immunol 2000, 164: $5120-5124$

19. Eil R, Vodnala SK, Clever D, Klebanoff CA, Sukumar M, Pan JH, Palmer DC, Gros A, Yamamoto TN, Patel SJ, Guittard GC, Yu Z, Carbonaro V, Okkenhaug K, Schrump DS, Linehan WM, Roychoudhuri R, Restifo NP: Ionic immune suppression within the tumour microenvironment limits T cell effector function. Nature 2016, 537:539-543

20. Tai HH, Tong M, Ding Y: 15-Hydroxyprostaglandin dehydrogenase (15-PGDH) and lung cancer. Prostaglandins Other Lipid Mediat 2007, 83:203-208

21. Rodriguez M, Clare-Salzler M: Eicosanoid imbalance in the NOD mouse is related to a dysregulation in soluble epoxide hydrolase and 15-PGDH expression. Ann N Y Acad Sci 2006, 1079:130-134

22. Bai Y, Wang J, He Z, Yang M, Li L, Jiang H: Mesenchymal stem cells reverse diabetic nephropathy disease via lipoxin A4 by targeting transforming growth factor $\beta$ (TGF- $\beta$ )/smad pathway and proInflammatory cytokines. Med Sci Monit 2019, 25:3069-3076 